\title{
A digital programmable telemetric system for recording extracellular action potentials
}

\author{
Francisco J. Heredia-López \\ Universidad Autónoma Metropolitana Iztapalapa, Mexico City, Mexico
}

José L. Bata-García, José L. Góngora-Alfaro, and Fernando J. Alvarez-Cervera

Universidad Autónoma de Yucatán, Mérida, Mexico

AND

JOAQUÍN AZPIROZ-LEEHAN

Universidad Autónoma Metropolitana Iztapalapa, Mexico City, Mexico

\begin{abstract}
This article describes the design and preliminary evaluation of a small-sized and low energy consumption wearable wireless telemetry system for the recording of extracellular neuronal activity, with the possibility of selecting one of four channels. The system comprises four radio frequency (RF) transceivers, three microcontrollers, and a digital amplifier and filter. This constitutes an innovative distributed processing approach. Gain, cutoff frequencies, and channel selection are remotely adjusted. Digital data transmission is used for both the bioelectrical signals and the control commands. This feature offers superior immunity to external RF interference. Real-time viewing of the acquired data allows the researcher to select only relevant data for storage. Simultaneous recordings of neuronal activity from the striatum of a freely moving rat, both with the wireless device and with a wired data acquisition system, are shown.
\end{abstract}

Recording neuronal activity in unrestrained animals has been one of the major goals in neuroscience, since this procedure allows patterns of neuronal firing to be correlated with specific behaviors. This has been successfully done with electronic devices in which the recorded neuronal activity is received through wires connected to electrodes implanted in discrete brain regions of the animals under study (Fee \& Leonardo, 2001). However, these systems can be used only with animals whose body movements have been restricted (Obeid, Nicolelis, $\&$ Wolf, 2004) or with small animals (e.g., birds, rats) that are free to move in small compartments. This hindrance has been overcome with the use of wireless telemetric devices (Eichenbaum, Pettijohn, Deluca, \& Chorover, 1977), which are more suited for studies in which neuronal activity should be recorded in animals able to move in complex environments (e.g., tunnels; Irazoqui, Mody, \& Judy, 2005) or over long distances (Lei et al., 2004).

Diverse strategies for sending neuronal electrical activity wirelessly have been developed. Until recently, all of these approaches used analog radio frequency (RF) links. Some of these prototypes are based on discrete components, whereas others use application-specific integrated circuits or hybrid configurations (Eichenbaum et al., 1977; Mohseni, Najafi, Eliades, \& Wang, 2005; Schregardus et al., 2006). Multichannel systems offer several advantages, such as the possibility of gathering data in less time, the opportunity to continue acquisition even when the signals from one or more electrodes are lost, the option of using stereotrodes, and the ability to compare traces obtained simultaneously from various neuronal structures (Liu, O'Connor, \& Strohl, 2006; Mohseni et al., 2005). Preliminary studies in which prerecorded data for digital processing and transmission have been used have been published as well (Bossetti, Carmena, Nicolelis, \& Wolf, 2004). A major advantage of digital data transmission is its superior immunity to external RF interference, as compared with that of analog systems. To our knowledge, so far, the only published work related to a telemetric system based on digital data transmission has been described by Obeid et al. (2004). However, this device, which was designed for experiments with monkeys, weighed $235 \mathrm{~g}$, making it unviable for use with smaller, unrestrained animals. Critical parameters for a biotelemetric system in small animals are high input impedance, minimal input noise, reduced voltage, low power consumption, compact size, and low weight. In addition, appropriate bandwidth and gain, among other electrical specifications, must be met, in accordance with the particular characteristics of neural signals (e.g., spike duration of about $1 \mathrm{msec}$ and amplitude on the order of $100 \mu \mathrm{V}$ ). 
Here, we present an alternative telemetric system for biopotential acquisition based on digital data transmission and distributed processing. It comprises three electronic units: a headstage, a backpack, and a base station connected to a personal computer $(\mathrm{PC})$. The headstage measures $2.0 \times$ $2.5 \times 2.4 \mathrm{~cm}$ and weighs $4.2 \mathrm{~g}$. The backpack, contained inside an enclosure placed on the back of the animal, measures $6.8 \times 4.7 \times 0.9 \mathrm{~cm}$ and weighs $10.5 \mathrm{~g}$, including the battery. Two reduced instruction set computer (RISC) type microcontrollers, two digital transceivers, and the required analog electronics are located inside this backpack. A 3-V battery powers this module. At the base station, a second pair of transceivers and a RISC-microcontroller-based interface for a PC completes the hardware. Communication between them is achieved through two bidirectional RF links, one of which is used for the digital transmission of the detected neuronal activity data, and the other to send the control commands for the remote unit. Specifically, this system allows changing the amplifier settings and filter parameters from the base station without disturbing the animal under study. Any one of the four recording channels can also be selected remotely. The tested range for fully reliable operation is $3 \mathrm{~m}$. The duration of the batteries $(\mathrm{Ni}$ Mh $170 \mathrm{mAh}$ ) under continuous operation is about $24 \mathrm{~h}$. The system has been evaluated on male Wistar rats, and a sample trace is shown. An additional feature under development is the possibility of repositioning the microelectrodes wirelessly by means of microstep motors mounted on a reusable custom microdrive.

\section{METHOD}

The headstage, as the initial component of the telemetric system, consists of the preamplifier stage and passive low-pass filters. It is connected to the backpack, which encompasses two sections: a bioelectric signal transmitter amplifier-filter and a command receiver for the control of the previous section and the future option of remote microelectrode repositioning. The first (signal transmission) section contains two programmable circuits (an am- plifier and an active band-pass filter), an RF transceiver, and a RISC-type microcontroller $(\mu \mathrm{C})$. The second (receiver) section includes a second RF transceiver, an additional RISC-type $\mu \mathrm{C}$, and a bipolar step motor driver. The base station includes two more transceivers and additional circuitry whose operation is directed by a more sophisticated RISC $\mu \mathrm{C}$. All electronic discrete and integrated components used in the prototype are surface-mounted devices. A general description of the circuit elements and the overall operation of the system follows.

\section{The Rat Module}

Headstage. The bioelectric signals can be obtained through up to four tungsten microelectrodes, one at a time. We found that the best recordings were obtained with electrodes having an impedance of $3.0 \mathrm{M} \Omega$ and a shaft diameter of $81 \mu \mathrm{m}$, including the parylene-C insulating material thickness (Model WE30033.0H3, Micro Probe, Gaithersburg, MD). A 1-mm-diameter stainless steel electrode was used as the voltage reference for the detected neuronal signals and was set to the midpoint of the power supply. For each of the four channels, which are transmitted individually, two INA2331 (Texas Instruments, Dallas, TX) dual-instrumentation amplifiers with an input polarization current specification of $0.5 \mathrm{pA}$ are used as high-input-impedance preamplifier stages for the microelectrode signals. In this case, they are wired with a fixed gain of 5 . The outputs of the preamps are connected to individual RC low-pass filters with a cutoff frequency of $10 \mathrm{kHz}$. The elements above, grouped in a single block, and the microelectrodes are shown in Figure 1.

Transmitter and programmable amplifier-filter. Figure 1 also shows the elements in the signal-processing path: a programmable gain amplifier (PGA), a programmable filter, an inverter output amplifier, a virtual ground generator, a RISC-type microcontroller (Microchip Technology, Chandler, AZ; Type PIC12F675; identified as Microcontroller 1) and a radio transceiver (DR3000-1; RF Monolithics, Dallas, TX; identified as Transmitter 1). Two PGA integrated circuits were used in the design of the am-

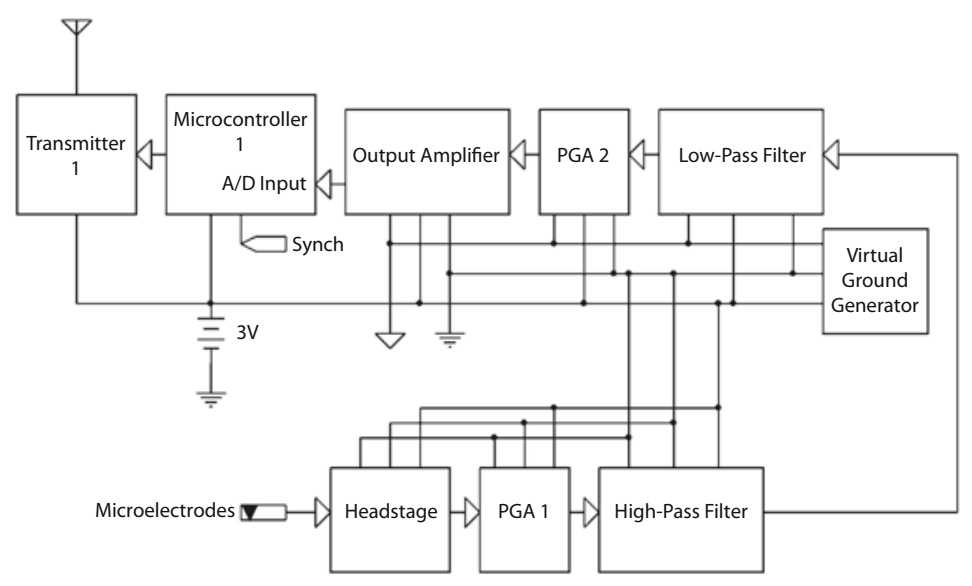

Figure 1. Signal path through the headstage, programmable gain amplifier (PGA), and transmitter section of the rat module. 


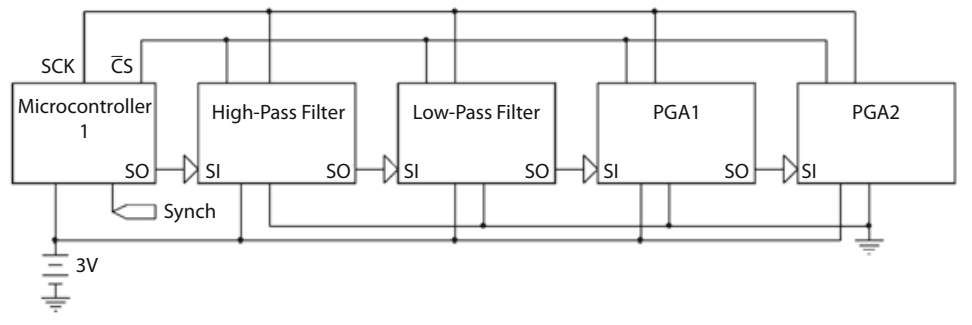

Figure 2. Programmable amplifier logic control chain. SCK, serial clock; CS, chip select; SO, serial data output; SI, serial data input. PGA, programmable gain amplifier.

plifier: an MCP6S26 with six multiplexed input channels and eight gain settings $(1,2,4,5,8,10,16$, and 32$)$, and an MCP6S21 with one input channel and the same eight gain values. Both PGAs are programmable using a serial protocol interface (SPI). They are connected in cascade to obtain 21 combined gain settings.

The programmable band-pass filter was designed with two MCP42050 (Microchip Technology) digital potentiometers, which are serially programmable using SPI and an MCP607 dual operational amplifier (Microchip Technology). The filter design is based on the classical SallenKey configuration for a voltage-controlled voltage source (VCVS) filter; by using the same component values and a DC gain of 1.59, a Butterworth filter is obtained. This stage contributes to the overall gain of the system with a 2.53 factor. The cutoff frequency of the high-pass programmable filter can be adjusted from 1.5 to $371 \mathrm{~Hz}$, whereas that of the low-pass filter can be selected from $68 \mathrm{~Hz}$ to $17.3 \mathrm{kHz}$.

One half of a second MCP607 in a voltage follower configuration is used to produce a virtual ground with its reference set to the midpoint of the $+3-\mathrm{V}$ power supply. This virtual ground is distributed throughout the circuits involved in signal processing. The second half of this dual amplifier is connected in an inverting configuration; it receives the output signal from the filter and amplifies it tenfold. It also acts as a low-output-impedance source for the analog-todigital converter (ADC) within Microcontroller 1. This is a high-input-impedance, rail-to-rail, output amplifier. This $\mu \mathrm{C}$ digitizes the output signal from the amplifier and, by means of the RS-232 serial protocol, sends the data bytes to Transmitter 1 (operating in transmitter mode) that is programmed for a $0.16-\mathrm{mW}$ output power, which is sufficient for a 3-m range. The maximum possible transmission range is specified by the manufacturer to be $100 \mathrm{~m}$ in an unobstructed open field. The range can be modified by changing the value of the modulating resistor, which determines the transmitter power. The data transmission rate is set at $125 \mathrm{~kb} / \mathrm{sec}$, which permits sending 21 data samples every $2 \mathrm{msec}$. It should be pointed out that although the manufacturer specification for this parameter is $115.2 \mathrm{~kb} / \mathrm{sec}$, we experimentally verified that the transmitter could operate at the higher rate of $125 \mathrm{~kb} / \mathrm{sec}$ without a decrease in performance. Samples consist of 8 bits of data plus 2 control bits. Thus, the word size is 10 bits, and the maximum transmission rate is $11.2 \mathrm{ksamples} / \mathrm{sec}$. This 8 -bit resolution is sufficient for spike detection and counting from a single channel, although it may not be enough for spike classification based on morphology.

Figure 2 shows the logic chain used to control the programmable system. As is illustrated, Microcontroller 1 manages the operation of the PGAs and the programmable filter (divided into high-pass and low-pass blocks), according to commands received through its synch pin. These instructions are issued by the second $\mu \mathrm{C}$ in the backpack unit (its operation is described later; it is identified as Microcontroller 2 in Figure 3).

Command receiver and controller center. This section uses a DR-3100 transceiver (RF Monolithics) in receiver mode (Receiver 1 in Figure 3). This device receives the incoming data in RS-232 serial format $(19.2-\mathrm{kb} / \mathrm{sec}$ baud rate, 8 data bits, no parity, 1 stop bit) and according to the experimental parameters selected by the user from the PC. This includes changing the gain, filter cutoff frequencies, and recording channel, as well as the movement commands for the step motor to be implemented in the future. These instructions are processed by the second PIC12F675 $\mu \mathrm{C}$ (Microcontroller 2 in Figure 3).

\section{The Base Station}

This module was designed around a PIC16F877 RISC $\mu \mathrm{C}$ (Microcontroller 3 ) and two transceiver circuits (a DR3000-1 and a DR-3100; see Figure 4). The DR3000-1 (operating in receiver mode and identified as Receiver 2) obtains the action potential information sent by the rat module (at $916.5 \mathrm{MHz}$ ). The serial output of this circuit is connected to an LM311 voltage comparator (National Semiconductor, Santa Clara, CA), whose function is to translate it from CMOS to TTL logical levels. These pulses are routed to the universal asynchronous receiver transmitter unit (UART) input of the $\mu \mathrm{C}$, where they are read and validated, then converted into parallel form, and finally transmitted to the $\mathrm{PC}$ via the parallel port, using the enhanced parallel port protocol. In case the user needs to change the experimental conditions (change the channel, gain, or filter cutoff frequencies, or move the step motor), the corresponding data are sent through the parallel port of the PC to the $\mu \mathrm{C}$, which then sends the information to the rat module, via RF.

\section{Software}

The PC program, written in LabVIEW 7.1 (National Instruments, Austin, TX), lets the user monitor the action 


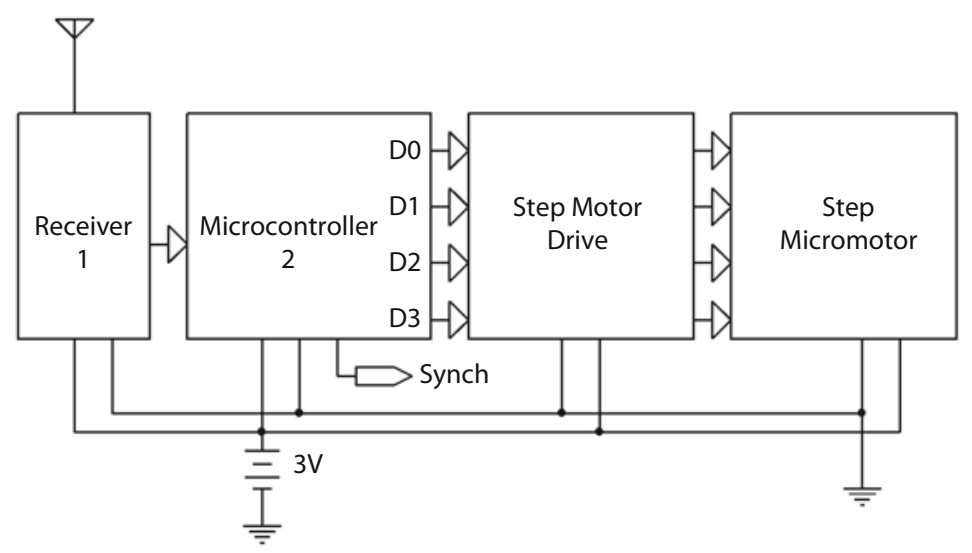

Figure 3. Command receiver and controller center (D0-D3 is the binary nibble used to move the step motor).

potentials at the time they are being detected; it permits saving useful data, as well as modifying the experimental conditions. The graphical display routine automatically scales the data plots according to the selected gain value.

The $\mu \mathrm{C}$ (Microcontroller 3 in Figure 4) program in the base station validates the $\mathrm{PC}$ commands and data to ensure that they were received error free. This program also adds two different sets of control bytes that, together with the one-byte code sent by the PC, increases to 24 the number of validation bits that are sent to Microcontroller 2 in the command receiver and controller center of the rat module.
This measure proved to be effective at eliminating the reception of incorrect experimental conditions or spurious motor movements.

The program in Microcontroller 2, in the command receiver and controller center, obtains the serial data from Receiver 1. It then analyzes the information and either resends the information to Microcontroller 1 in the transmitter and programmable amplifier-filter or uses it directly to move the step motor. The program in Microcontroller 1 of the transmitter programmable amplifierfilter receives data from Microcontroller 2 and uses it to select the proper channel, the corresponding gain of the

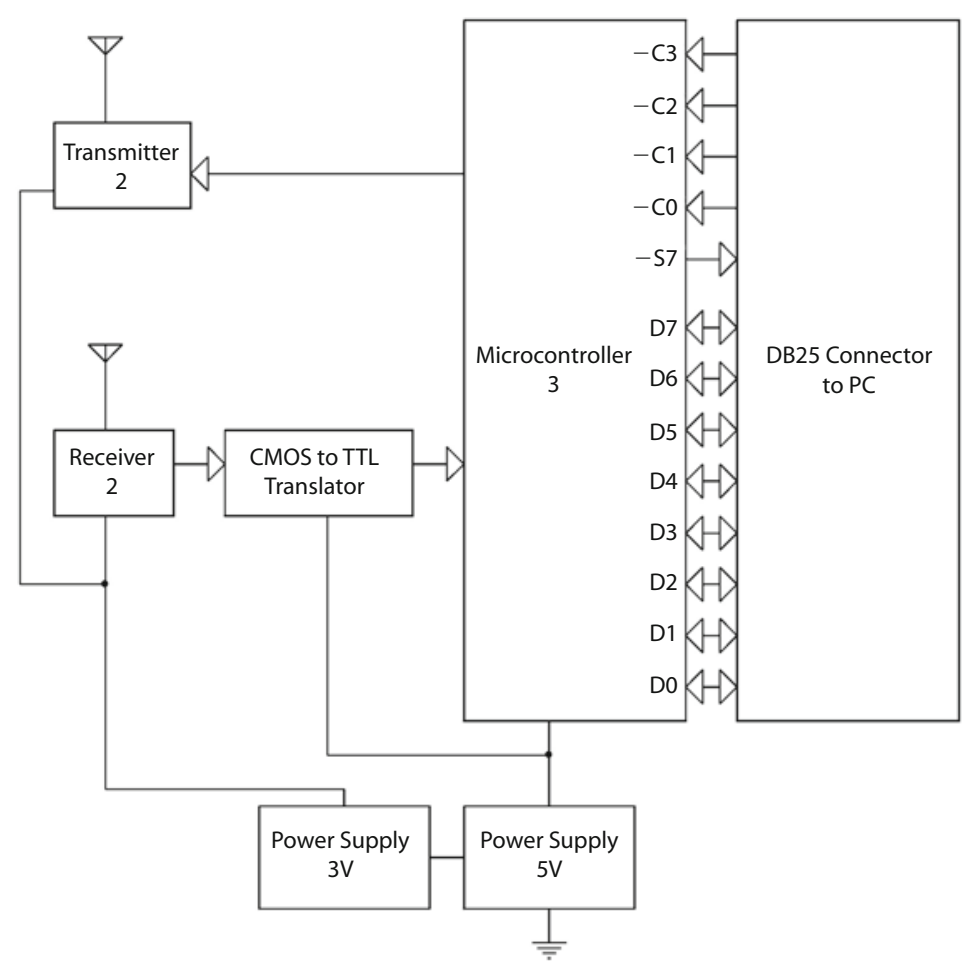

Figure 4. Base station (D0-D7 is the PC parallel data port, $\mathrm{CO}-\mathrm{C} 3$ is the $\mathrm{PC}$ parallel control port, $\mathrm{S} 7$ is one bit from the $\mathrm{PC}$ parallel status port). 


\section{A}
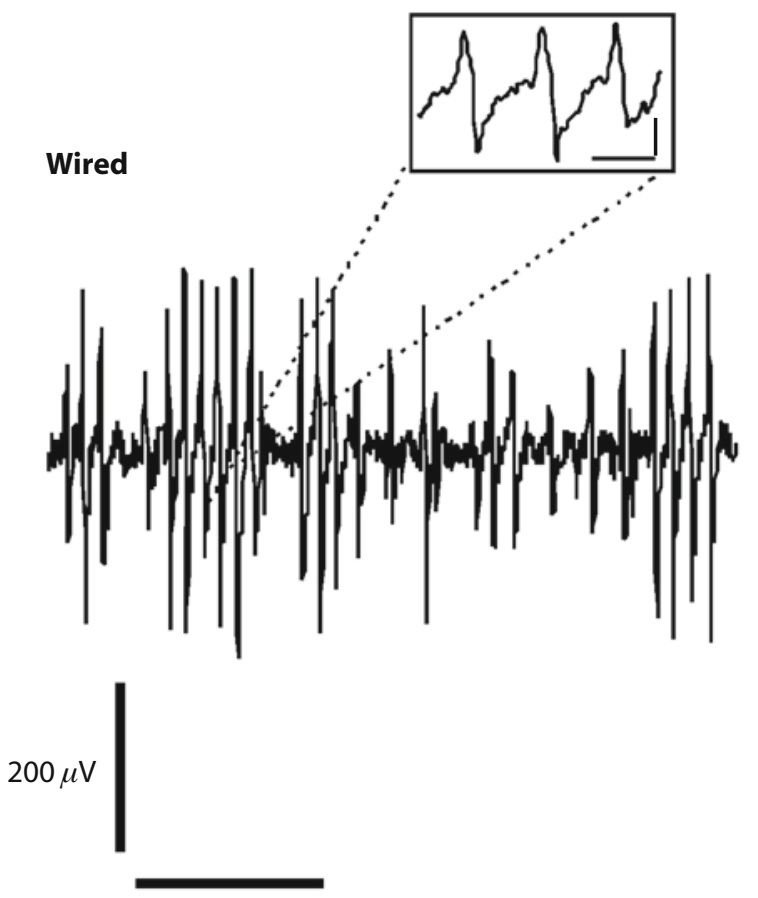

$50 \mathrm{msec}$

\section{B}

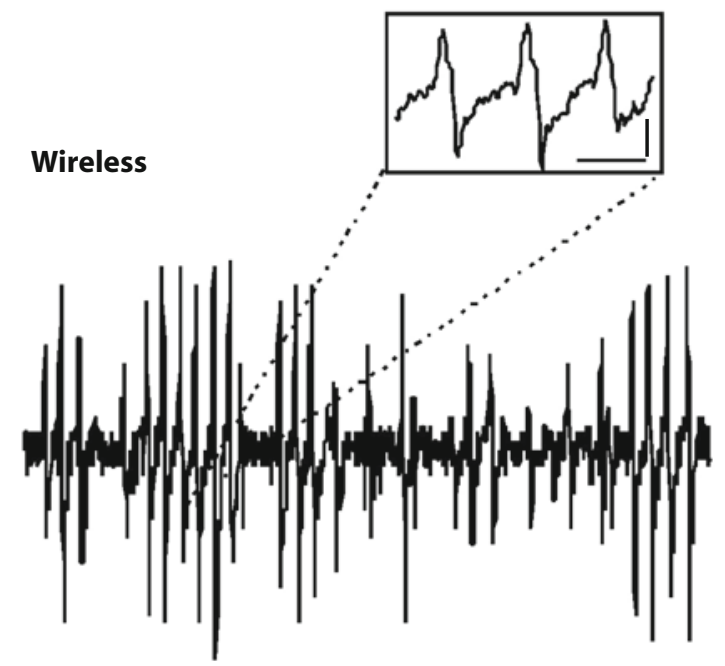

Figure 5. (A) Electrical activity from the striatum of a fully awake rat obtained with a battery-operated DAM 80 commercial amplifier, and with the following conditions: gain of 5000, filters programmed for $300 \mathrm{~Hz}$ in the low-frequency cutoff and $3000 \mathrm{~Hz}$ in the high-frequency end. (B) Trace obtained with the telemetric system with a gain of 5060 , a low cutoff frequency of $386 \mathrm{~Hz}$, and a high cutoff frequency of $3087 \mathrm{~Hz}$. The time scale bars for both inserts are 2 msec.

amplifier, and the frequency band of the filters, using the SPI protocol.

\section{Experimental Data Collection}

For the in vivo tests, 6 male adult Wistar rats $(270$ $310 \mathrm{~g}$ in weight) bred in our facilities were employed. All efforts were made to minimize animal suffering according to the recommendations of the Guide for the Care and Use of Laboratory Animals (National Research Council, 1996). They were anesthetized with sodium pentobarbital $(45 \mathrm{mg} / \mathrm{kg}$, i.p.) and were placed in a stereotaxic frame (Stöelting) equipped with a manual microdrive, with the incisor bar set $3.3 \mathrm{~mm}$ below the interaural line. Tungsten microelectrodes $(3.0 \mathrm{M} \Omega)$ were directed to the right striatum at the following coordinates: AP, $+1.0 \mathrm{~mm}$ from bregma; $\mathrm{L},-3.5 \mathrm{~mm}$ from the midline; and V, $-4.3 \mathrm{~mm}$ from the dura surface (Paxinos \& Watson, 1986), in a region associated with motor control (Chang, Wachtel, Young, \& Kang, 1999). The recording sessions were performed 7 days after surgery, with the fully awake rat implanted with the microdrive headstage and the telemetric device. The animals were placed in a cage, and stable recordings were obtained during $3-4 \mathrm{~h}$ and were performed with the complete setup placed inside a Faraday cage with a mesh wire spacing of about $2 \mathrm{~cm}$, which is appropriate for blocking $60-\mathrm{Hz}$ interference. Recordings may be made without the use of a Faraday cage, although the amount of interference will depend on the presence of electrical devices or networks in the vicinity. To verify the fidelity of signal transmission, simultaneous recordings were made in the striatum of awake rats with a commercial batterypowered amplifier, using a wired connection, as well as with the developed wireless telemetric system.

\section{RESULTS}

Figure 5A shows a 500-msec sample of the neuronal activity of the striatum from an awake rat obtained with a commercial amplifier. Comparisons of the electrical activity in the rat brains were made with the simultaneous recordings from the telemetric system and from a conventional commercial bioelectric amplifier. In order to keep the electrical isolation of the telemetric system and avoid the interference due to electrical noise, a battery-operated DAM 80 (World Precision Instruments) extracellular recording amplifier was used, with a gain of 5,000 and filters programmed for $300 \mathrm{~Hz}$ in the low cutoff and $3000 \mathrm{~Hz}$ in the high cutoff frequencies. The signal output of this amplifier was connected to an NI-USB-6009 data acquisition module (National Instruments Corp.), which was USB-linked to a laptop PC running on battery energy. In Figure $5 \mathrm{~B}$, the same signal transmitted and recorded simultaneously with the telemetric system is shown. A gain of 5,060 was used, and the filters were programmed for $386 \mathrm{~Hz}$ in the low cutoff frequency and $3087 \mathrm{~Hz}$ in the high-frequency end. The average SNR for the case of the telemetric system is about 4.4, whereas for the commercial wired system, it is around 4.5. For both approaches, the sampling rate was set to 11,200 samples per second. Figure 6 shows a rat implanted with the headstage. The headstage measures $2.0 \times 2.5 \times 2.4 \mathrm{~cm}$, weighs $4.2 \mathrm{~g}$, and includes the space that will be occupied by the micromotor and additional mechanical components to be added in the future in order to displace the microelectrodes through wireless control. In fact, the headstage shown contains an acrylic dummy weight in this space. 


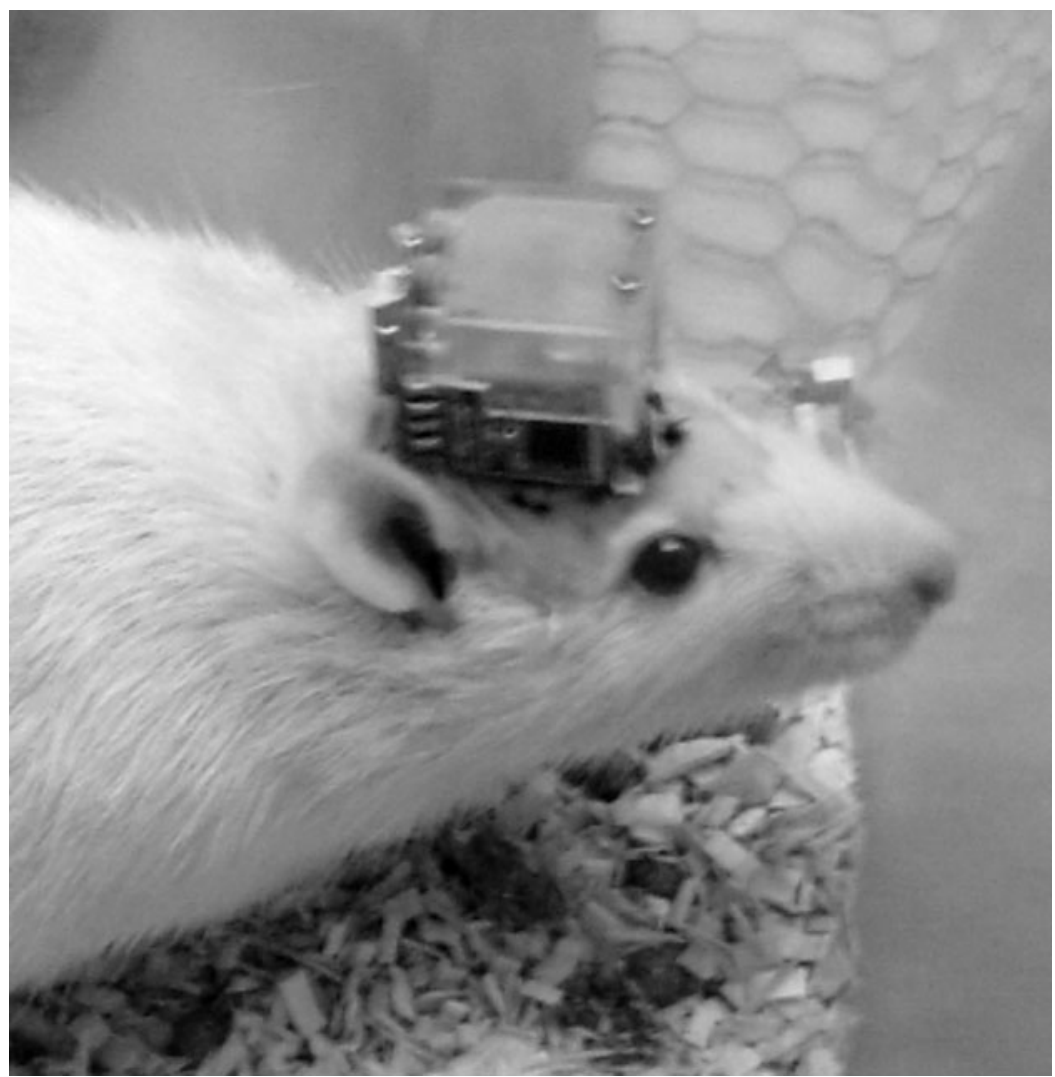

Figure 6. Rat implanted with the headstage. The headstage measures $2.0 \times 2.5 \times$ $2.4 \mathrm{~cm}$ and weighs $4.2 \mathrm{~g}$.

\section{DISCUSSION}

As is shown in the results above, the system is capable of detecting neural activity in the rat brain and transmitting it wirelessly without a significant loss of information. The described unit allows the experimenter to remotely change the single channel to be recorded, as well as to modify the amplifier gain setting and the filter cutoff frequencies. These critical adjustments can be made without perturbing the animal. Due to the limitations of the bandwidth of the RF transmitter and the digitization speed of the ADC converter, only one channel at a time can be transmitted with this prototype. The present system is designed for use in freely moving animals and for the future addition of a microelectrode-repositioning feature. Since the mechanical components occupy an important part of the headstage, there is limited space for additional electronics and electrodes.

However, the importance of this design is that, sometimes, during long-term studies, electrodes can stop recording electrical activity due to cell death or to the loss of mechanical contact. In this case, the adjacent electrodes can still be used to record the same type of activity at nearby regions. The electrode-repositioning system will improve this capability, in addition to allowing the study of the electrical activity at different layers in the brain structures.
Once neuronal activity has been detected, amplification and filtering parameters may need to be modified from the previous settings for optimal signal-to-noise ratios. Again, these adjustments can be accomplished in the described system without immobilizing the animal or altering its behavior in any way. The signal-to-noise ratio of the obtained recordings is about 4.4 , which is comparable to the values reported for analog telemetric systems (Lei et al., 2004; Nieder, 2000; Pinkwart \& Borchers, 1987).

Digital data transmission is an important feature used in the present approach and has the inherent advantage of reduced susceptibility to ambient noise. Having the data in digital form simplifies the storage, display, exchange, and analysis of experimental information in a PC. In particular, digital data is more suitable for the implementation of spike detection and sorting algorithms (Obeid et al., 2004). Furthermore, this system permits the visualization of recordings in real time, allowing the researcher to monitor the progress of the recording session and to select only relevant data for storage. An automated scheme for electrophysiological signal recording also allows the researcher to concentrate on studying behavioral aspects during the experiments. It is important to note that the relatively high power consumption $(10.86 \mathrm{~mA})$ of this prototype, as compared with other telemetric systems (Chien \& Jaw, 2005; Takeuchi \& Shimoyama, 2004), is due to the use of two 
continuous closed-loop transceivers. One of them, permanently acting as a command receiver, utilizes about $4.5 \mathrm{~mA}$, whereas the second one, configured as a transmitter, uses approximately $6 \mathrm{~mA}$. The rest of the electronic components require less than $1 \mathrm{~mA}$. This level of current consumption led to the use of a relatively large battery (220-mA capacity and $9-\mathrm{g}$ weight), and with the future implementation of a remote-controlled motorized microdrive, an even larger power source will be required. Still, the total weight for the backpack reported here $(10.5 \mathrm{~g})$ is tolerable for a rat, and even with a more powerful battery (such as a CR2477 $3 \mathrm{~V} \mathrm{Li} \mathrm{cell),} \mathrm{the} \mathrm{increase} \mathrm{in} \mathrm{weight} \mathrm{would} \mathrm{be} \mathrm{only} 4 \mathrm{~g}$. However, this apparent disadvantage is highly compensated for by the versatility obtained by being able to remotely select the experimental conditions for a single channel at a time and by the superior performance of the digital approach. The battery duration is greater than $24 \mathrm{~h}$, and the tested transmission range was $3 \mathrm{~m}$.

As has already been indicated, work is under way to incorporate a motorized microdrive for electrode repositioning, also under telemetric control. Both the hardware and software components of this feature have been considered in the current design, and only the microdrive development is pending.

In conclusion, the present system offers the following salient features: distributed data and task processing for an enhanced performance, remote control of experimental conditions (channel, gain, filtering) to avoid any animal manipulation, digital information exchange (data and commands), adequate range for open-field studies in rats $(3 \mathrm{~m})$, small size $\left(2.7-\mathrm{cm}^{3}\right.$ headstage; $28.8-\mathrm{cm}^{3}$ backpack), and low weight ( 2.2 and $10.5 \mathrm{~g}$, respectively). Moreover, the flexibility conferred by the modular design and by the programmability permits the use of the system with other physiological signals (ECG, EEG, EMG, respiration, etc.) by changing only the preamplifier section and through minor changes in the PC software.

\section{AUTHOR NOTE}

This work was sponsored by SEP-CONACyT-México Grant 200301-31-001-056 (2003-2006) to F.J.H.-L. We are indebted to Gloria Arankowsky-Sandoval and Angel Saúl Castilla-Tirado for their invaluable technical assistance, and to Alberto Caballero-Ruiz and Leopoldo Ruiz-Huerta for their useful advice regarding the future motorized microdrive implementation. The authors declare that they have no competing financial interests. Correspondence concerning this article should be addressed to F. J. Alvarez-Cervera, Departamento de Neurociencias, Centro de Investigaciones Regionales "Dr. Hideyo Noguchi," Universidad Autónoma de Yucatán, Mérida, Mexico (e-mail: acervera@uady.mx).

\section{REFERENCES}

Bossetti, C. A., Carmena, J. M., Nicolelis, M. A. L., \& Wolf, P. D. (2004). Transmission latencies in telemetric-linked brain-machine interface. IEEE Transactions on Biomedical Engineering, 51, 919-924.

Chang, J. W., Wachtel, S. R., Young, D., \& Kang, U. J. (1999). Biochemical and anatomical characterization of forepaw adjusting steps in rat models of Parkinson's disease: Studies on medial forebrain bundle and striatal lesions. Neuroscience, 88, 617-628.

Chien, C. N., \& JAW, F. S. (2005). Miniature telemetry system for the recording of action and field potentials. Journal of Neuroscience Methods, 147, 68-73.

Eichenbaum, H., Pettijohn, D., Deluca, A. M., \& Chorover, S. L. (1977). Compact miniature microelectrode-telemetry system. Physiology \& Behavior, 18, 1175-1178.

Fee, M. S., \& Leonardo, A. (2001). Miniature motorized microdrive and commutator system for chronic neural recordings in small animals. Journal of Neuroscience Methods, 112, 83-94.

IRAZOQUI, P. P., ModY, I., \& JUDY, J. W. (2005). Recording brain activity wirelessly: Inductive powering in miniature implantable neural recording devices. IEEE Engineering in Medicine Biology Magazine, 24, 48-54.

LeI, Y., Sun, N., Wilson, A. W., WANG, X., Chen, N., YANG, J., ET AL. (2004). Telemetric recordings of single neuron activity and visual scenes in monkeys walking in an open field. Journal of Neuroscience Methods, 135, 35-41.

Liu, C.-C., O'Connor, E., \& Strohl, K. P. (2006). A multichannel, wireless telemetric microsystem for small animal studies. IEEE Sensors Journal, 6, 187-202.

Mohseni, P., Najafi, K., Eliades, S., \& Wang, X. (2005). Wireless multichannel biopotential recording using an integrated FM telemetry circuit. IEEE Transactions on Neural Systems \& Rehabilitation Engineering, 13, 263-271.

National Research Council (1996). Guide for the care and use of laboratory animals. Washington, DC: National Academy Press.

NiEDER, A. (2000). Miniature stereo radio transmitter for simultaneous recording of multiple single-neuron signals from behaving owls. Journal of Neuroscience Methods, 101, 157-164.

Obeid, I., Nicolelis, M. A. L., \& Wolf, P. D. (2004). A multichannel telemetry system for single unit neural recordings. Journal of Neuroscience Methods, 133, 33-38.

PaXINOS, G., \& WaTson, W. (1986). The rat brain in stereotaxic coordinates (2nd ed.). San Diego: Academic Press.

Pinkwart, C., \& Borchers, H. W. (1987). Miniature three-function transmitting system for single neuron recording, wireless brain stimulation and marking. Journal of Neuroscience Methods, 20, 341-352.

Schregardus, D. S., Pieneman, A. W., Maat, A. T., Jansen, R. F., Brouwer, T. J. F., \& GAHR, M. L. (2006). A lightweight telemetry system for recording neuronal activity in freely behaving small animals. Journal of Neuroscience Methods, 155, 62-71.

TaKeuchi, S., \& Shimoyama, I. (2004). A radio-telemetry system with shape memory alloy microelectrodes for neural recording of freely moving insects. IEEE Transactions on Biomedical Engineering, 51, 133-137.

(Manuscript received September 12, 2008; revision accepted for publication December 9, 2008.) 